Paper:

\title{
Determination of Aspect Ratio Limitations, Accuracy and Repeatability of a Laser Line Scanning CMM Probe
}

\author{
Bart Boeckmans*, Min Zhang ${ }^{*, * *}$, Frank Welkenhuyzen*, and Jean-Pierre Kruth* \\ * Department Mechanical Engineering, Division Production Engineering, Machine Design and Automation, KU Leuven \\ Celestijnenlaan 300, B-3001 Leuven, Belgium \\ E-mail: Bart.Boeckmans@kuleuven.be \\ ${ }^{* *}$ Beijing Engineering Research Center of Precision Measurement Technology and Instruments, \\ Beijing University of Technology (BJUT) \\ Beijing 100124, China \\ [Received March 31, 2015; accepted May 31, 2015]
}

\begin{abstract}
Coordinate measurement machine (CMM) probing techniques can involve direct mechanical contact (e.g., tactile probing) or diverse non-contact principles (e.g., laser line scan probing). For some applications, contact methods are not capable of measuring fast enough to ensure $100 \%$ quality controlled parts. A laser line scanning probe uses a laser triangulation-based method to acquire 3D measurement points on a workpiece relative to a sensor. Mounting the sensor in a 3D coordinate frame, e.g., in a CMM provides enough information to fully examine the workpiece. These techniques are most commonly exploited in medical industry and industries involving plate materials. A high data density and measurement speed are significant advantages when measuring free-form surfaces by laser line scanning, making the process much more time-efficient. However, high-precision geometrical features (such as cylinders, spheres, etc.) must be measured for locating and aligning the free-form shapes. The accuracy of the equipment therefore has to be assessed. Probe Maximum Permissible Error $\left(\mathrm{MPE}_{\mathrm{P}}\right)$ values below $10 \mu \mathrm{m}$ have been reported for cutting-edge laser line scanners. This paper compares the major influences on measurements on cylindrical features. First, the aspect-ratio limitations are considered by comparing two inherently different techniques. The stable inspection of reference features is important, while trying to maximize the spatial extent of the measured features. Second, the measurement method is analyzed in two ways: by using a limited sample of the features to increase stability and eliminate interference from neighboring features; by varying the number of scan tracks, which greatly affects the measurement time.
\end{abstract}

Keywords: dimensional metrology, CMM, laser line scanning, accuracy, repeatability

\section{Introduction}

This study focuses on coordinate measurement machine (CMM) techniques where quality control (calibration, verification, and traceability) is paramount [1-6]. Tactile probe measurements are usually taken as the reference in industry, because they are reliable and well understood. Tactile probes are used in CMMs but also for onmachine product measurements and machine error verification $[7,8]$. The measurement speed is usually a limiting factor. Moreover, laser line scanning, a triangulationbased optical measurement method, has also been described in the literature [9]. The data acquisition speed of a laser line scanner is much greater (up to 75,000 points per second in comparison to up to 2 points per second for touch-trigger probing). In this technique, the sensor can be implemented in either arm-based [10] or coordinatebased (CMM) [9] measurement frame. The use of these different probes in the same application can combine their advantages [11-15]. The main benefits of laser line scanning are its high acquisition speed, its high data density, and its independence of the form to be measured. The latter signifies that multiple features can be scanned simultaneously, and that even free-form surfaces can be assessed quickly and compared to a CAD model. However, one drawback of this approach is its sensitivity to reflective surface properties and the large number of operator settings. Surface properties (e.g., texture and reflectivity) strongly influence both the measurement offset with respect to a tactile reference value $[16,17]$ and the quality of measurement $[12,18]$.

Figure 1a shows the general structure of the laser line scanning probe. The angle $\gamma$ defines the opening between the plane of laser light and the center viewing direction. This fixed parameter is one of the determining factors of the maximum measurable aspect ratio (Section 4). Another important restriction is the field of view (FOV, Fig. 1b), which describes the extent of the intersection of the camera view with the laser plane $[9,13]$.

Recently, laser line scanners evolved into sensors that can achieve maximum permissible errors for the probe $\left(\mathrm{MPE}_{\mathrm{P}}\right)$ below $10 \mu \mathrm{m}$. Accuracy tests have been de- 


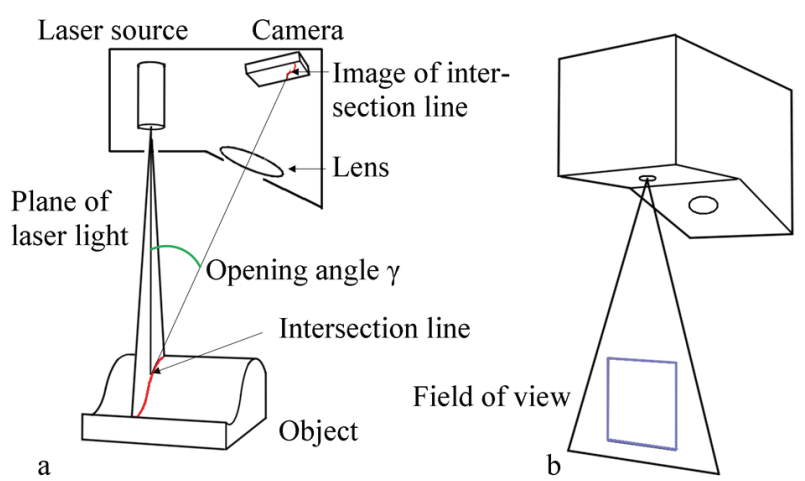

Fig. 1. Laser line scanning probe: (a) the sensor structure and (b) the field of view (FOV) limitation [9].

veloped $[9,19-21]$ and the operation of optical CMM probes [22-24] has become more standardized. A standard has been introduced to verify the $\mathrm{MPE}_{\text {System }}$ for a CMM equipped with an optical probe [25].

This paper presents an overview of the equipment and then discusses the measurement procedures. Subsequently it investigates the factors that affect the achievable measurement depth for cylindrical holes. Lastly, it considers an industrial case study to test the findings and to appraise the accuracy and the repeatability of two CMM probes.

\section{Equipment and Materials}

\subsection{Measuring Equipment}

The measurements presented in this paper were taken with two CMMs: an LK Altera and a Coord3 MC16 CMM. The corresponding $\mathrm{MPE}_{\mathrm{CMM}}$ specifications are given, respectively, as:

$$
\begin{aligned}
& \mathrm{MPE}_{\mathrm{LK}}=1.8+\frac{L}{400} \quad \ldots . \quad . \quad . \quad . \quad . \quad . . \\
& \mathrm{MPE}_{\mathrm{Coord} 3}=5+\frac{L}{200} \quad \text {. . . . . . . . . }
\end{aligned}
$$

with the measured length $L$ expressed in millimeters and MPE in micrometers. One touch-trigger probe (Renishaw TP200) and one optical probe (Nikon Metrology LC60Dx laser line scanner) are used. The specified MPEP value for the latter is $9 \mu \mathrm{m}$ (in accordance with ISO 103601 [26] and ISO 10360-8 [25]). The probes are linked to the CMM through a Renishaw PH10M rotary head.

\subsection{Artefacts and Case Study Objects}

The reference artefact used in this study (Fig. 2a) is a ground prismatic object with twelve drilled and reamed cylindrical holes of various diameters $(1-20 \mathrm{~mm})$. The main purpose was to verify the maximum measurable aspect ratio (Section 4) of this set of cavities. The industrial case study objects (Fig. 2b) are a pair of machine parts, each with a different surface finish, that also include cylindrical features. These two objects were used to investigate
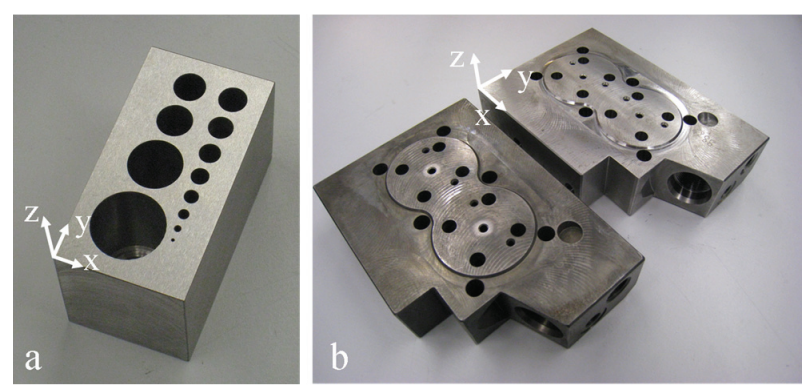

Fig. 2. Measurement objects: (a) reference artefact with holes of various size, and (b) matte and shiny industrial case study objects.

the effect of different surface reflections and measurement strategies, as explained in Section 5.

\section{Measurement Procedures}

The repeatability and accuracy results reported in Section 5 are based on a single tactile measurement approach (reference measurement) and four distinct methods for the optical laser line scan probe measurements.

The tactile reference measurement was performed in a straightforward manner, with the stylus orientation approximately parallel to the cylinder axes. Thirty two points were probed, covering a range of four depth levels.

In a laser line scan with the sensor set to this orientation, very few measurement points fall onto the internal cylinder walls; the projected laser plane is nearly parallel to the cylinder wall and the reflected intensity toward the capturing sensor is extremely low. Therefore, the in-plane angle $\alpha$ of the sensor [9] (i.e., the angle with respect to the normal of the upper plane) was set to a non-zero value. To have a balanced surface coverage, two opposite (e.g., $0^{\circ}$ and $180^{\circ}$ ) or four quadrant-spread (e.g., $-90^{\circ}, 0^{\circ}, 90^{\circ}$ and $180^{\circ}$ ) out-of-plane angles were selected to scan the inner surfaces of the cavities. Another option is to use either the full acquired dataset of the cylinder wall or a limited subset measured along the cylinder axis. These two surface-coverage settings (two or four out-of-plane scanning angles; full versus restricted axial section) yield a $2 \times 2$ design of experiments (DoE) matrix. This will be discussed in Section 5.3.

\section{Maximum Measurable Aspect Ratio}

The limitations on the aspect ratio can be considered as follows. The maximum measurable aspect ratio is the ratio between the maximum depth for which a cylinder diameter can be assessed adequately, given a set of measurement settings and the feature size. The experimental results from this section were obtained utilizing the Altera CMM. A detailed discussion can be found in [27]. 


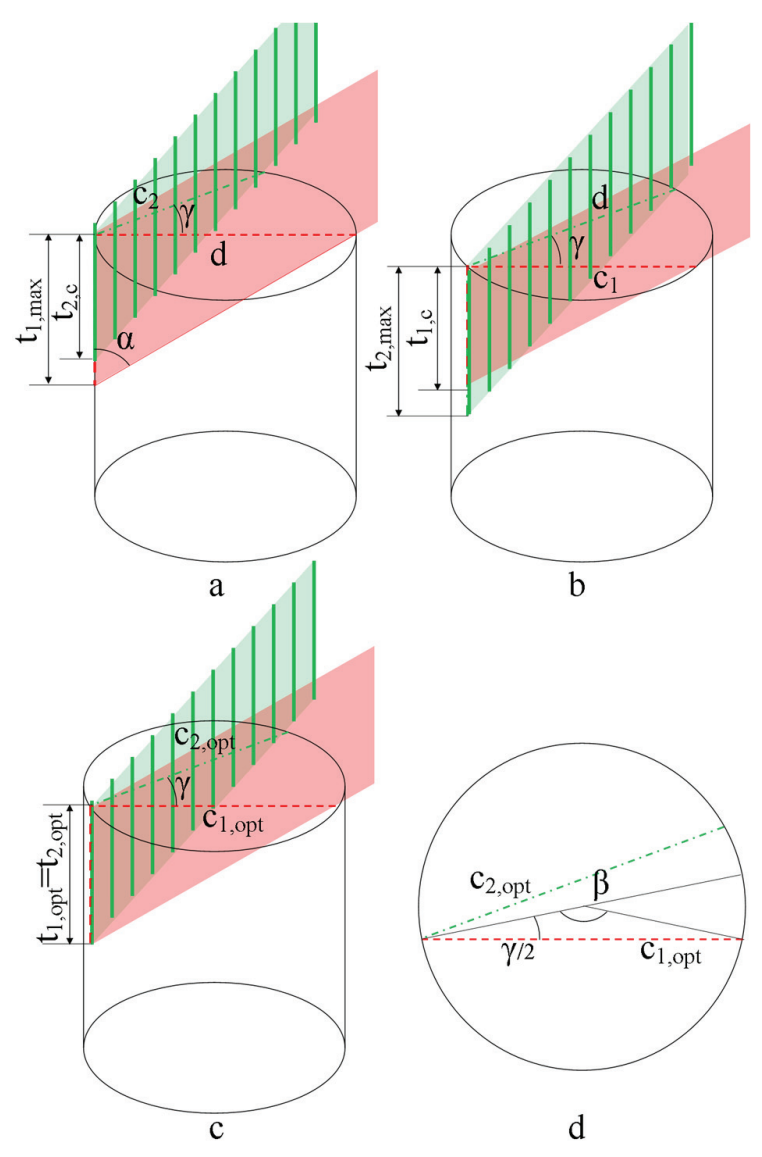

Fig. 3. Measurement scenarios with (a) maximum laser depth $t_{1, \max }$, (b) maximum viewing depth $t_{2, \max }$, (c) optimal combination $t_{1, \mathrm{opt}}=t_{2, \mathrm{opt}}$. (d) Top view of the scenario in (c), showing the optimal chord length $\left(c_{\mathrm{opt}}\right)$.

\subsection{Theoretical Analysis}

The maximum measurable aspect ratio can be derived as an optimal condition (see Fig. 3c). In Figs. 3a-d, the extreme and optimal situations are depicted, using a fully shaded red plane for the projected laser plane and a green striped plane for the incident camera viewing mid plane. The angle between the two planes is the opening angle $\gamma$ in the worst-case scenario. Fig. 3a shows the situation where the laser plane coincides with the maximum chord length on the circular top section, i.e., spanning the full diameter $d$. The depth down to which the laser plane penetrates into the cylinder is denoted as $t_{1, \max }$ and can be calculated with Eq. (3), using the tangent of the in-plane angle setting $\alpha$ as a conversion factor. However, this is deeper than the camera view can access, being limited to a chord length $c_{2}$.

$$
c=t \cdot \tan \alpha
$$

The set of conditions illustrated in Fig. 3b show a very similar situation, although the viewing depth is maximal here while the laser light penetration depth is suboptimal. Both of these scenarios occur at different times. However, a globally optimal intermediate scenario exists, wherein both the laser light plane and the camera view are individ-

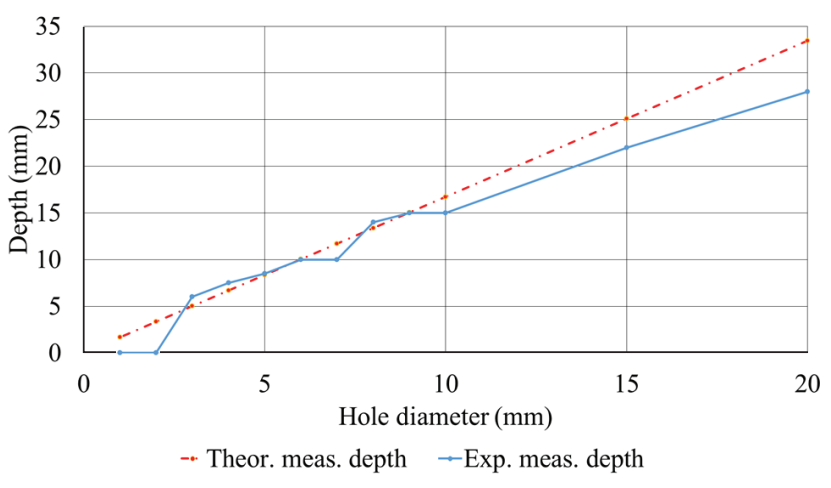

Fig. 4. Theoretical and experimental measurement depths, plotted as functions of the hole diameter.

ually suboptimal but overlap maximally.

This optimal situation is illustrated in Fig. 3c from a 3D perspective and in Fig. 3d from a top-view perspective. The same chord length $c_{\mathrm{opt}}$ is subtended in the topview circular section for both the projected light and the captured light. This chord length can be determined from Eq. (4) in terms of the diameter and the inscribed angle $\beta$, which itself depends on the angle between the projected laser plane and the viewing direction. In the worst case, this angle equals the opening angle $\gamma$.

$$
c=d \cdot \sin \left(\frac{\beta}{2}\right)=d \cdot \sin \left(\frac{180-\gamma}{2}\right) \ldots .
$$

By combining Eqs. (3) and (4), the theoretical maximum measurable depth can be determined. Using this depth and the diameter of the cavity, the theoretical maximum measurable aspect ratio can also be calculated.

\subsection{Results and Discussion}

Figure 4 compares plots of the experimental and theoretical results for the maximum measurable depth of cylindrical features. The results are shown for an in-plane incident angle $\alpha$ of $30^{\circ}$ (sensor orientation with respect to the normal of the top plane). The experimental results were acquired by scanning the entire object and doing fittings on the resulting point cloud. The search distance for the fitted points was limited to a 4-mm-deep section. The depth into the cavity was gradually increased until stability was no longer guaranteed. A good correlation can be found for the middle section of the graph (diameters 3-10 mm). For smaller diameter values, the experimental results deviate from the theoretical values because the amount of data is too limited to perform a correct fitting. For holes larger than the FOV of the laser line scanner (diameters $>15 \mathrm{~mm}$ ), the data points at the bottom of the hole are incorrect because the physical aspect ratio of the cylindrical cavities is too low. Direct and multiple reflections from the bottom surface can produce scatter points that compromise the correctness of the measurement at the bottom of the cylindrical feature.

Figure 5 plots one of the cylinder parameters (the $x$ position - see Fig. 2a) derived from a tactile and an opti- 


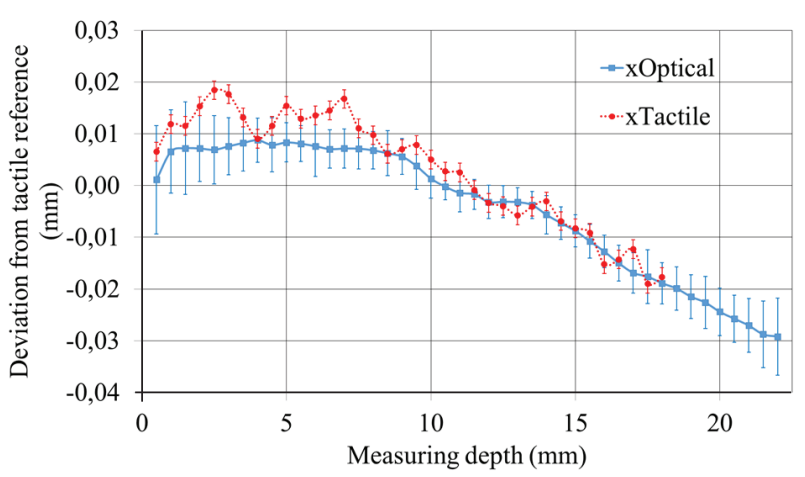

Fig. 5. Deviations from the full cylinder tactile reference value for the $x$ position for the optical and tactile measurement, as functions of the measurement depth.

cal measurement, as a function of the measurement depth. The reference (zero value) in Fig. 5 is the tactile measurement of the entire cylinder (maximum axial coverage). Two observations can be made from this graph. First, the $x$ position of the hole shifts when moving deeper into it, indicating an inclination of the cavity. There is a linear shift of approximately $35 \mu \mathrm{m}$ over a depth range of 5 to $20 \mathrm{~mm}$. Second, the tactile measurement shows more variation than the optical measurement. Although both graphs are well correlated, an important difference is the smaller range of the tactile measurement compared to the optical measurement, which makes it more sensitive to local deviations in the cavity wall.

\section{Industrial Case Study}

\subsection{Case Study Objective and Description}

The goal of this case study is to verify the capabilities of the laser line scanner in terms of the maximum measurable aspect ratio and the scanner's repeatability and accuracy. As the two industrial parts considered have a different surface finish, the impact of surface conditions and of the measurement strategy on the measurement result can be investigated. These case study objects are depicted in Fig. 2b. This study focused on the $8.5-\mathrm{mm}$ cylindrical cavities.

The aim of the first set of tests (Section 5.2) is to verify the capabilities of the scanner and to provide insight into the factors that influence the measurements. For these experiments, an LC60Dx laser line scanner and a TP200 probe were compared on an Altera CMM. The resulting data sets were acquired in two series of measurements with the non-contact equipment. The first series used a quick approach (involving two opposite scanning angles), taking into account the entire point cloud; the second used a slower scanning approach (with four angles arranged in quadrants) using only a limited subset of the acquired data points. Preliminary tests provided insight into the other sensor settings. First, an in-plane angle $\alpha$ of $30^{\circ}$ gave the best compromise between the scannable depth and sta-

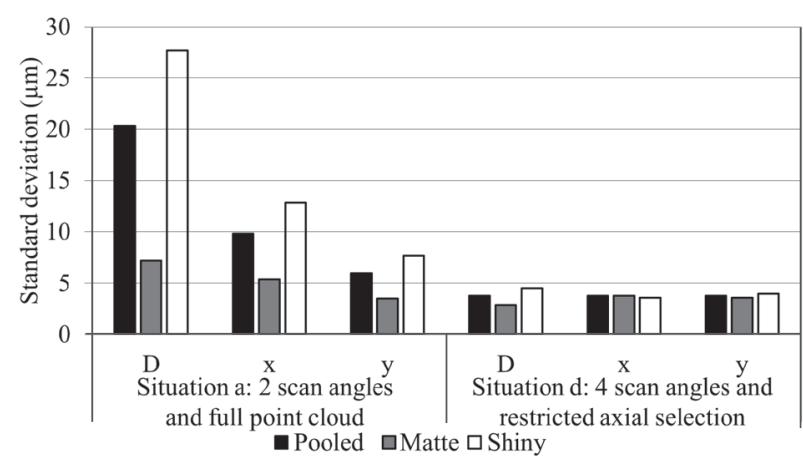

Fig. 6. Comparison of the combined (pooled) and separate (based on the surface finish) standard deviations, obtained by the two measurement methods [27].

bility in the measurement points acquired [27]. Second, the earlier described tests show an optimal position for the limited data sample, located below the top surface at a depth ranging from 7 to $15 \mathrm{~mm}$ for an 8-mm diameter hole (Section 4.2). Both industrial parts (matte and shiny) were measured. Nine repeated measurements were done for all six of the 8.5-mm diameter cavities. This provided enough data for a statistical analysis. The measured parameters were the cavity diameter and the cavity position (Fig. 2b, $x$ and $y$ coordinates).

The second part of the investigations (Section 5.3) served to confirm the findings of the first set and to gain more detailed insight into the relative significance of the influence factors. The tests were conducted on a Coord3 MC16 CMM using an LC60Dx probe. Four scan variations were used:

(a) two scan angles, using the full point cloud;

(b) two scan angles, using a restricted axial selection;

(c) four scan angles, using the full point cloud;

(d) four scan angles, using a restricted axial selection.

Only the industrial part with the shiny surface was considered for this section. The measurement settings and the number of repetitions were kept constant. The reference values were unchanged (TP200 measurement on the Altera CMM).

\subsection{Comparing Repeatability and Accuracy}

Figure 6 compares the repeatability and the accuracy of the two highest-contrast approaches (situations $a$ and $d$ ). This graph distinguishes between the two case study objects. An average value and a standard deviation were calculated for all shiny and matte surface finish features (each comprising 54 measurements and 48 degrees of freedom). The statistical pooled standard deviations for the combined matte and shiny results are also shown. Two main conclusions can be drawn.

First, the approach involving the four quadrant-spread scanning angles and a restricted axial selection from the 


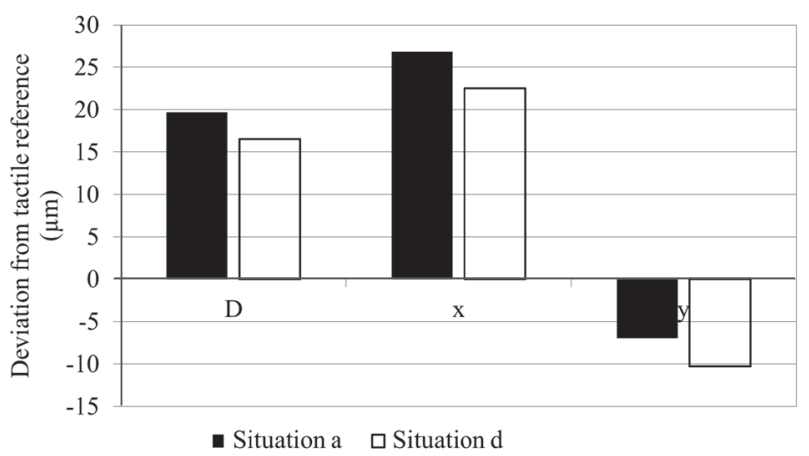

Fig. 7. Comparison of the deviations of the two optical measurements relative to the tactile measurement, for a feature on the matte case study object [27].

point cloud yields a lower overall standard deviation. All but one variable (the $y$ position for the matte surface) improve under these scanning conditions. Both parameters appear to be beneficial to the standard deviation of the measurement. The use of four scanning angles implies that a greater proportion (close to $100 \%$ ) of the circumference of the cylinder surface is assessed, which increases the stability when moving and rotating the sample on the CMM between consecutive iterations. The restricted axial selection avoids contaminating the fitting algorithm with scatter points from the bottom surface and additional noisy measurement points from the top surface. The relative importance of the two parameters is explored further in Section 5.3.

The second observation is the difference between the matte- and the shiny-surface results. The matte industrial object seems to be less sensitive to the above-mentioned effects, despite showing a small overall improvement.

The accuracy results for one cavity are depicted in Fig. 7. Both methods yield very similar average values (for nine measured sample values), with differences of between 3 and $10 \mu \mathrm{m}$. The accuracy seems to be only weakly influenced by the parameter variations.

\subsection{Discriminating the Influence Factors}

As shown in Fig. 7 and discussed in Section 5.2, there is a small difference between the average deviations from the tactile reference measurement, which influences the accuracy of the assessment. Additional experiments conducted using all four optical approaches yielded similar values (Fig. 8). Differences in the range 4-16 $\mu \mathrm{m}$ were recorded. Results for another sample, measured with a different machine (the Coord3 MC 16 CMM), are shown for the set of repeated measurements. The observed larger values can be attributed to the higher values for the CMM

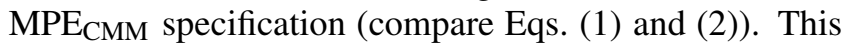
would only result in modest visible changes for the diameter and $x$ position assessment. The $y$ position shows a larger shift that can be linked to an error in the measurement process, during the alignment procedure for the last measurement set. The plane restricting the $y$ position was assessed differently because of coordinate shifts for

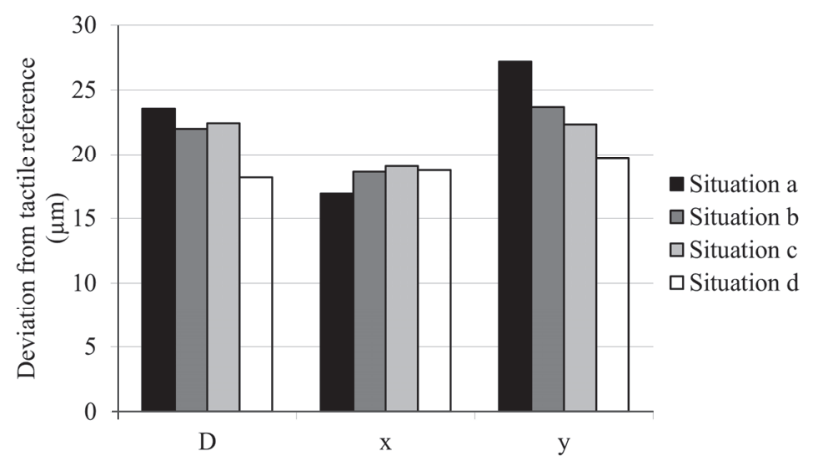

Fig. 8. Comparison of the measurement deviations, relative to the tactile reference, for the extended set of measurement approaches on the shiny industrial case study object.

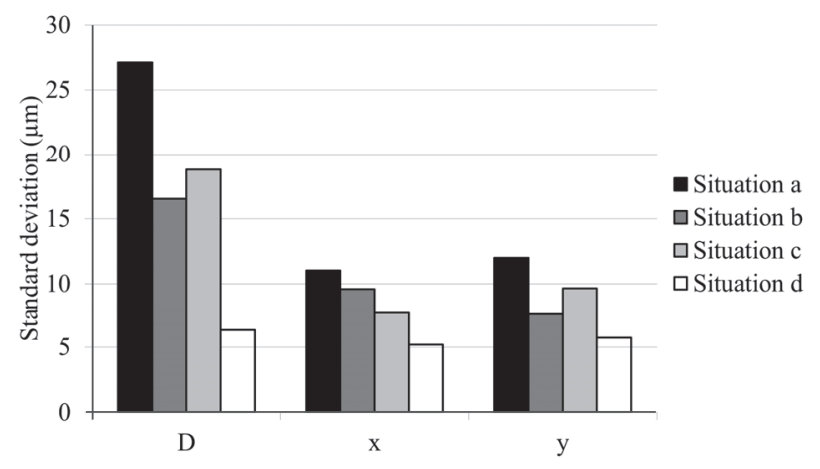

Fig. 9. Comparison of standard deviations for the extended set of measurement approaches on the shiny industrial case study object.

six of the twelve measurement points. This resulted in an increase in the $y$ position for the entire workpiece.

Figure 9 represents a sample comparable to those in Fig. 6, but now including all four optical-measurement approaches. Note again that only the shiny case study object was assessed in all four cases. Although the standard deviations seem to show an overall increase, both figures display common trends.

First, the CMM in the second tests again is attributed a worse MPE compared to the first CMM, which may explain the overall rise in both error to the tactile reference and standard deviation.

Second, there is a clear difference between situations $a$ and $d$, which confirms the results on the LK Altera CMM described in Section 5.2.

A third observation is that the less stable situation (i.e., with a higher standard deviation) shows a more pronounced difference for the diameter measurement than for the position measurements.

Finally, we attempted to discriminate the relative importance of the influencing factors. These results only indicate that both parameters have a similar impact on the standard deviation of the optical measurement with the laser line scanner. Neither of the two parameters is significantly more influential. 


\section{Conclusion}

This study sought to contribute to a better understanding of the laser line scanner, used as a CMM probe. Several influence factors were considered. First, we discussed the maximum measurable aspect ratio. Theoretical predictions and experimental results were correlated, and their discrepancies explained. Second, we investigated the impact of surface conditions. Shiny surfaces are more prone to instability when the fitting algorithm covers a wider search field and when using fewer scanning angles. Thirdly, we tried to discriminate the relative impact of using a limited cylinder section and scanning with fewer sensor orientations. We found a consistent increase in the measurement standard deviation when using fewer scanning angles and when using a full cylinder fitting, rather than when using more scanning angles and a limited cylinder section fitting. Both parameters seem to contribute with similar orders of magnitude.

\section{Acknowledgements}

This study was funded by the agentschap voor Innovatie door Wetenschap en Technology (IWT) through the Tetra project Multisensor coördinaten meettechniek voor snel en accuraat opmeten van complexe producten (MuSeS - 120167).

\section{References:}

[1] S. Osawa, T. Takatsuji, T. Kurosawa, R. Furutani, and M. Shibata, "Traceability system of coordinate measuring machines through internet," J. of the Japan Society for Precision Engineering, Vol.70, No.4, pp. 528-532, 2004.

[2] K. Umetsu, R. Furutani, S. Osawa, T. Takatsuji, and T. Kurosawa, "Optimization of measurement strategy for calibration of coordinate measuring machine," J. of the Japan Society for Precision Engineering, Vol.70, No.9, pp. 1174-1179, 2004.

[3] Y. Asano, R. Furutani, and M. Ozaki, "Verification of interim check method of CMM," Int. J. of Automation Technology, Vol.5, No.2, pp. 115-119, 2011.

[4] J. Matsuda, "Uncertainty analysis of three-dimensional coordinate measuring machines," J. of Robotics and Mechatronics, Vol.9, No.2, pp. 140-145, 1997.

[5] Y. Takaya, "In-process and on-machine measurement of machining accuracy for process and product quality management: a review," Int. J. of Automation Technology, Vol.8, No.1, pp. 4-19, 2014.

[6] O. Jusko, M. Neugebauer, H. Reimann, and R. Berhardt, "Recent progress in CMM-based form measurement," Int. J. of Automation Technology, Vol.9, No.2, pp. 170-175, 2015.

[7] S. Ibaraki and Y. Ota, "Error calibration for five-axis machine tools by on-the-machine measurement using a touch-trigger probe," Int. J. of Automation Technology, Vol.8, No.1, pp. 20-27, 2014.

[8] Y. Ihara and T. Nagasawa, "Fundamental study of the on-machine measurement in the machining center with a touch trigger probe," Int. J. of Automation Technology, Vol.7, No.5, pp. 523-536, 2013.

[9] N. Van Gestel, S. Cuypers, P. Bleys, and J.-P. Kruth, "A performance evaluation test for laser line scanners on CMMs," Optics and Lasers in Engineering, Vol.47, pp. 336-342, 2009.

[10] H. Hamana, M. Tominaga, M. Ozaki, and R. Furutani, "Calibration of articulated arm coordinate measuring machine considering measuring posture," Int. J. of Automation Technology, Vol.5, No.2, pp. 109-114, 2011.

[11] A. Weckenmann, X. Jiang, K.-D. Sommer, U. Neuschaefer-Rube, J. Seewig, L. Shaw, and T. Estler, "Multisensor data fusion in dimensional metrology," CIRP Annals - Manufacturing Technology Vol.58, No.2, pp. 701-721, 2009

[12] H. Zhao, J.-P. Kruth, B. Boeckmans, N. Van Gestel, and P. Bleys, "Automated dimensional inspection planning using the combination of laser scanner and tactile probe," Measurement, Vol.55, No.5, pp. 1057-1066, 2012.

[13] A. Contri, P. Bourdet, and C. Lartigue, "Quality of 3D digitized points obtained with non-contact optical sensors," CIRP Annals Manufacturing Technology, Vol.51, No.1, pp. 443-446, 2002.
[14] C. Lartigue, A. Contri, and P. Bourdet, "Digitised point quality in relation with point exploitation," Measurement, Vol.6, pp. 193-203, 2002.

[15] H. Feng, Y. Liu, and F. Xi, "Analysis of digitizing errors of a laser scanning system," Precision Engineering, Vol.25, pp. 185-191, 2001.

[16] Y. Tan, "Scanning and post-processing parameter optimization for CT dimensional metrology," Ph.D. Thesis, Katholieke Universiteit Leuven, Department of Mechanical Engineering, 2015 (in press).

[17] B. Boeckmans, Y. Tan, F. Welkenhuyzen, Y. S. Guo, W. Dewulf, and J.-P. Kruth, "Roughness offset differences between contact and non-contact measurements," Proc. of Euspen 15 ${ }^{\text {th }}$ Int. Conf., 2015, in press.

[18] B. Igor, N. Van Gestel, J.-P. Kruth, P. Bleys, and J. Hodolič, "Accuracy improvement of laser line scanning for feature measurements on CMM," Optics and Lasers in Engineering, Vol.45, No.11, pp. 1274-1211, 2011

[19] A. Bernard and M. Véron, "Analysis and Validation of 3D Laser Sensor Scanning Process," CIRP Annals - Manufacturing Technology, Vol.48, No.1, pp. 111-114, 1999.

[20] B. Boeckmans, F. Welkenhuyzen, and J.-P. Kruth, "Accuracy verification of a laser line scanner probe," Laser Metrology and Machine Performance X, pp. 279-288, 2013.

[21] B. Boeckmans, M. Zhang, F. Welkenhuyzen, W. Dewulf, and J.-P. Kruth, "CMM sensor evaluation method with machine error exclusion," Laser Metrology and Machine Performance XI, 2015.

[22] ISO 14253-1:1998. GPS - Inspection by measurement of workpieces and measuring equipment - Part 1: Decision rules for proving conformance or non-conformance with specifications.

[23] ISO 14253-2:2011, GPS - Inspection by measurement of workpieces and measuring equipment - Part 2: Guide to the estimation of uncertainty in GPS measurement, in calibration of measuring equipment and in product verification.

[24] ISO, GUM, JCGM 100:2008: Evaluation of measurement data Guide to the expression of uncertainty in measurement.

[25] ISO 10360-8:2013. Geometrical Product Specifications (GPS) Acceptance and reverification test for coordinate measuring machines (CMM) - Part 8: CMMs with optical distance sensors.

[26] ISO 10360-1:2000, Geometrical Product Specifications (GPS) Acceptance and reverification tests for coordinate measuring machines (CMM) - Part 1: Vocabulary.

[27] B. Boeckmans, M. Zhang, F. Welkenhuyzen, W. Dewulf, and J.-P. Kruth, "Comparison of aspect ratio, accuracy and repeatability of a laser line scanning probe and a tactile probe," Proc. of the $11^{\text {th }}$ IMEKO Symposium (LMPMI2014), A14, 2014.

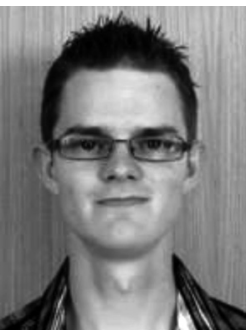

Name:

Bart Boeckmans

\section{Affiliation:}

Ph.D. Researcher, Department Mechanical Engineering, Division Production Engineering, Machine Design and Automation, KU Leuven

Address:

Celestijnenlaan 300, 3001 Leuven, Belgium

Brief Biographical History:

2010-2012 Scientific Coworker at KU Leuven

2012- Ph.D. Researcher, Department Mechanical Engineering, Division Production Engineering, Machine Design and Automation, KU Leuven Main Works:

- "Visual Inspection of powder deposition in Selective Laser Melting," Machine Vision and Applications, Springer International, 2011.

- "Accuracy verification of a laser line scanner probe," Laser Metrology and Machine Performance X, pp. 279-288, 2013.

- "Comparison of Aspect Ratio, Accuracy and Repeatability of a Laser Line Scanning Probe and a Tactile Probe," 10th IMEKO Symposium: Laser Metrology for Precision Measurement and Inspection in Industry (LMPMI), A14, 2014. 


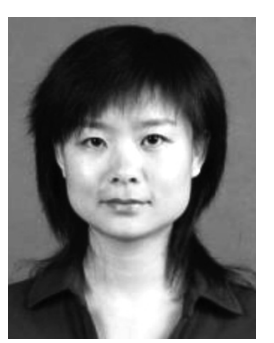

Name:

Min Zhang

\section{Affiliation:}

Research Associate, Catholic University of Leuven (KU Leuven)

Lecturer and Supervisor, Beijing University of Technology

Address:

Celestijnenlaan 300, 3001 Leuven, Belgium

Brief Biographical History:

2008-2011 Ph.D. in Applied Sciences - Mechanical Engineering, École Normale Superieure de Cachan

2012- Lecturer, Beijing University of Technology

2014-2015 Research Associate, Catholic University of Leuven (KU

Leuven)

Main Works:

- Over 10 publications in journals and proceedings

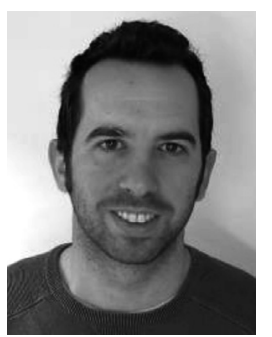

Name:

Frank Welkenhuyzen

\section{Affiliation:}

Ph.D. Researcher, Department Mechanical Engineering, Division Production Engineering, Machine Design and Automation, KU Leuven

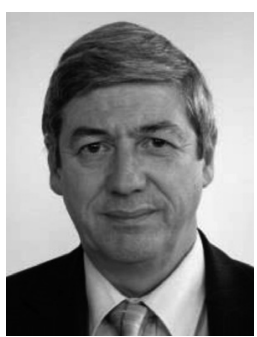

Name:

Jean-Pierre Kruth

\section{Affiliation:}

Full Professor and Research Team Leader, Department Mechanical Engineering, Division Production Engineering, Machine Design and $\mathrm{Au}-$ tomation (PMA), KU Leuven

Address:

Celestijnenlaan 300, 3001 Leuven, Belgium

Brief Biographical History:

1979 Received Ph.D. in Applied Sciences - Mechanical Engineering from KU Leuven

1979-1982 Lecturer, Institut Technologi Bandung, Indonesia

1983-1987 Senior Research Engineer, WTCM-CRIF (Research Centre of Belgian Metalworking Industry)

1987- Professor, Catholic University of Leuven (KU Leuven)

Main Works:

- Over 500 publications in journals and proceedings

- Industrial and Academic Career Award in Virtual and Rapid Prototyping (Portugal, 2009)

- International (Senior) Freeform and Additive Manufacturing Excellence Award (FAME, Austin, USA, 2012)

- SME Albert M. Sargent Progress Award (USA, 2014)

- Laureate of the Franklin Institute (Bower Science Award, 2015)

Membership in Academic Societies:

- International Academy for Production Engineering (CIRP), Fellow

- Society of Manufacturing Engineers (SME), Fellow

- International Measurement Confederation (IMEKO), Member

\section{Address:}

Celestijnenlaan 300, 3001 Leuven, Belgium

\section{Brief Biographical History:}

2007- Scientific Coworker, KU Leuven

2012- Ph.D. Researcher, Department Mechanical Engineering, Division

Production Engineering, Machine Design and Automation, KU Leuven

\section{Main Works:}

- "Industrial computer tomography for dimensional metrology: Overview of influence factors and improvement strategies," Proc. of the 4th Int.

Conf. on optical measurement techniques for structures and systems, 2009.

- "Accuracy study of a $450 \mathrm{kv}$ ct system with a calibrated test object,"

Proc. of the 11th Int. Symposium on Measurement and Quality Control (ISMQC-2010), 2013.

- "Investigation of the kinematic system of a $450 \mathrm{kv}$ ct scanner and its influence on dimensional ct metrology applications," Proc. of the Int. Conf. on Industrial Computed Tomography, 2014.

- "Simulation of x-ray projection images for dimensional ct metrology," Proc. of the 5th Int. Conf. on optical measurement techniques for structures and systems, 2012.

- "Accuracy enhancement of the measuring probe for a camera based mobile CMM," LAMDAMAP 2009, Teddington, UK, June 30 to July 2 , 2009. 